\title{
MARTINGALE CONVERGENCE TO INFINITELY DIVISIBLE LAWS WITH FINITE VARIANCES
}

BY

\author{
B. M. BROWN AND G. K. EAGLESON
}

\begin{abstract}
Some results are obtained concerning the convergence in distribution of the row sums of a triangular array of certain dependent random variables. The form of dependence considered is that of martingales within rows, and the results are obtained under conditions which parallel those of the classical case of convergence in distribution, to infinitely divisible laws with bounded variances, of the row sums of elementary systems of independent random variables.
\end{abstract}

1. Introduction. The problem of the convergence in law of row sums of elementary systems of independent random variables (r.vs.) was solved by Bavli's introduction [1] of the idea of accompanying laws. In this paper, we use an analogue of the accompanying laws in an attempt to extend the classical results for independent r.vs. to certain classes of dependent r.vs., which we call martingale elementary systems.

In $\S 2$ we obtain sufficient conditions (exactly analogous to the classical conditions) for the convergence in distribution of the row sums of martingale elementary systems to infinitely divisible laws with finite variances.

2. A limit law for martingale elementary systems. Consider a double array of random variables, which we take without loss of generality to be a triangular array, whose rows are martingale difference sequences, i.e. for each $n=1,2, \ldots$, we have r.vs. $X_{n 1}, \ldots, X_{n n}$ on a probability space $(\Omega, \mathscr{F}, P)$, with sub $\sigma$-fields $\mathscr{F}_{n 0} \subset \mathscr{F}_{n 1} \subset \ldots \subset \mathscr{F}_{n n}$ of $\mathscr{F}$ such that $X_{n k}$ is $\mathscr{F}_{n k}$-measurable and $E\left(X_{n k} \mid \mathscr{F}_{n, k-1}\right)=0$ almost surely (a.s.) for $k=1,2, \ldots, n$. Such arrays will be called martingale triangular arrays. Let

$$
\begin{aligned}
S_{n n} & =X_{n 1}+\cdots+X_{n n}, & \sigma_{n k}^{2} & =E\left(X_{n k}^{2} \mid \mathscr{F}_{n, k-1}\right), \\
V_{n k}^{2} & =\sum_{j=1}^{k} \sigma_{n j}^{2}, & b_{n} & =\max _{j \leqq n} \sigma_{n j .}^{2} .
\end{aligned}
$$

We will assume that

$$
b_{n} \stackrel{p}{\rightarrow} 0 \text { as } n \rightarrow \infty \text {, }
$$

Received by the editors September 16, 1970.

AMS 1970 subject classifications. Primary 60F05; Secondary 60E05, 60G45.

Key words and phrases. Triangular arrays, martingale elementary system, accompanying laws, infinitely divisible laws with bounded variances, martingale differences, conditional expectations. 
and that there exists a finite constant $C$ for which

$$
\lim _{n \rightarrow \infty} P\left(V_{n n}^{2}>C\right)=0 \text {. }
$$

If the r.vs. $X_{n 1}, \ldots, X_{n n}$ are independent, then $V_{n n}^{2}$ is a.s. constant and equal to $E S_{n n}^{2}$. Equations (1) and (2) then constitute the requirements for the triangular array to form an elementary system (see Gnedenko [4, p. 316]). We define a martingale triangular array to be a martingale elementary system if its rows are martingale difference sequences which obey equations (1) and (2).

THEOREM 1. Assume that there exists a bounded nondecreasing function $G(\cdot)$ for which

$$
\sum_{k=1}^{n} E\left(X_{n k}^{2} I\left(a<X_{n k} \leqq b\right) \mid \mathscr{F}_{n, k-1}\right) \stackrel{p}{\longrightarrow} G(b)-G(a)
$$

as $n \rightarrow \infty$, for all continuity points $a, b$ of $G$. Then $S_{n n}$ converges in law as $n \rightarrow \infty$ to an infinitely divisible limit law whose characteristic function (ch.f.) $\phi(\cdot)$ is given by

$$
\log \phi(t)=\int_{-\infty}^{\infty}\left(e^{i t x}-1-i t x\right) x^{-2} d G(x) .
$$

The proof of the theorem is based on a technique of Lévy which was later used by Billingsley [2], and in [3]. In essence it consists of finding a martingale sum $S_{n}^{*}$ which is close to $S_{n n}$ in some sense, but which is sufficiently well behaved to yield a limit law without difficulty.

The following routine lemma will be needed in the proof.

Lemma. Let $X_{n}, W_{n}, n=1,2, \ldots$, be r.vs., $f(t)$ a characteristic function and, for some fixed $t$, assume that $W_{n}^{-1}$ converges to $(f(t))^{-1}$ in the mean of order 1 , and that

$$
\lim _{n \rightarrow \infty} E\left(W_{n}^{-1} \cdot \exp \left(\text { it } X_{n}\right)-1\right)=0 .
$$

Then

$$
\lim _{n \rightarrow \infty} E \exp \left(\text { it } X_{n}\right)=f(t)
$$

Proof.

$$
\begin{aligned}
\left|E\left(\exp \left(i t X_{n}\right)-f(t)\right)\right| & \leqq\left|E \exp \left(i t X_{n}\right)\left(1-W_{n}^{-1} f(t)\right)\right|+\mid E f(t)\left(W_{n}^{-1} \exp \left(\text { it } X_{n}\right)-1\right) \mid \\
& \leqq E\left|1-W_{n}^{-1} f(t)\right|+|f(t)| \cdot \mid E\left(W_{n}^{-1} \exp \left(\text { it } X_{n}\right)-1\right) \mid \\
& \leqq E\left|W_{n}^{-1}-(f(t))^{-1}\right|+\left|E\left(W_{n}^{-1} \exp \left(i t X_{n}\right)-1\right)\right| \\
& \rightarrow 0 \text { as } n \rightarrow \infty
\end{aligned}
$$

Proof of the theorem. For each fixed $n$, let $X_{n k}^{*}=X_{n k} I\left(V_{n k}^{2} \leqq C\right)$, for $k=1,2, \ldots$, $n$. $\left\{X_{n 1}^{*}, \ldots, X_{n n}^{*}\right\}$ forms a martingale difference sequence since $I\left(V_{n k}^{2} \leqq C\right)$ is $\mathscr{F}_{n, k-1^{-}}$ measurable for all $k=1,2, \ldots, n$. If we write $V_{n k}^{* 2}=\sum_{i=1}^{k} E\left(X_{n i}^{* 2} \mid \mathscr{F}_{n, i-1}\right)$ then

$$
V_{n n}^{* 2} \leqq C \text { a.s. for all } n=1,2, \ldots
$$


Moreover, from (2) we have

$$
\lim _{n \rightarrow \infty} P \bigcap_{j=1}^{n}\left[X_{n j}=X_{n j}^{*}\right]=1,
$$

so that (3) holds with $X_{n j}$ replaced by $X_{n j}^{*}, j=1,2, \ldots, n$. Because of (6), it will suffice to prove Theorem 1 with $X_{n j}^{*}$ replacing $X_{n j}, j=1, \ldots, n$, enabling use to be made of the property (5). Alternatively, we will prove the theorem as it stands and assume also that

$$
V_{n n}^{2} \leqq C \quad \text { a.s., } \quad \text { all } n=1,2, \ldots
$$

For fixed $t$, let $g_{t}(x)=\left(e^{i t x}-1-i t x\right) x^{-2}$. For arbitrary positive $\varepsilon_{1}, \varepsilon_{2}$ and $\varepsilon_{3}$, choose an integer $m$ sufficiently large and a subdivision $x_{0}<x_{1}<\cdots<x_{m}$, all continuity points of $G$, so that

$$
\begin{gathered}
\left|\int_{-\infty}^{\infty} g_{t}(x) d G(x)-\sum_{j=1}^{m} g_{t}\left(x_{j-1}\right)\left(G\left(x_{j}\right)-G\left(x_{j-1}\right)\right)\right|<\varepsilon_{1}, \\
\max _{j \leqq m}\left(x_{j}-x_{j-1}\right)<\varepsilon_{2}, \quad\left|g_{t}(x)\right|<\varepsilon_{3} \quad \text { for } x \leqq x_{0} \text { or } x>x_{m} .
\end{gathered}
$$

Then, using (7), it follows that

$$
\sum_{k=1}^{n} \sum_{j=1}^{m} g_{t}\left(x_{j-1}\right) E\left(X_{n k}^{2} I\left(x_{j-1}<X_{n k} \leqq x_{j}\right) \mid \mathscr{F}_{n, k-1}\right)
$$

differs from

$$
\sum_{k=1}^{n} \sum_{j=1}^{m} E\left(g_{t}\left(X_{n k}\right) X_{n k}^{2} I\left(x_{j-1}<X_{n k} \leqq x_{j}\right) \mid \mathscr{F}_{n, k-1}\right)
$$

by less than $M_{t} \varepsilon_{2} C$ (where $M_{t}=\sup _{x}\left|d g_{t}(x) / d x\right|<\infty$ ) and that the latter term differs from $\sum_{k=1}^{n} E\left(g_{t}\left(X_{n k}\right) X_{n k}^{2} \mid \mathscr{F}_{n, k-1}\right)$ by less than $\varepsilon_{3} C$. Finally, from (3) we have

$$
\begin{aligned}
& \sum_{k=1}^{n} \sum_{j=1}^{m} g_{t}\left(x_{j-1}\right) E\left(X_{n k}^{2} I\left(x_{j-1}<X_{n k} \leqq x_{j}\right) \mid \mathscr{F}_{n, k-1}\right) \\
& \stackrel{p}{\longrightarrow} \sum_{j=1}^{m} g_{t}\left(x_{j-1}\right)\left(G\left(x_{j}\right)-G\left(x_{j-1}\right)\right) \quad \text { as } n \rightarrow \infty .
\end{aligned}
$$

Combining all information from equation (8) onwards we obtain

$$
\sum_{k=1}^{n} E\left(g_{t}\left(X_{n k}\right) X_{n k}^{2} \mid \mathscr{F}_{n, k-1}\right) \stackrel{p}{\rightarrow} \int_{-\infty}^{\infty} g_{t}(x) d G(x)
$$

as $n \rightarrow \infty$, for all fixed $t$. But

$$
\left|g_{t}(x) \cdot x^{2}\right| \leqq \frac{1}{2} t^{2} x^{2}
$$

so that

$$
\left|\sum_{k=1}^{n} E\left(g_{t}\left(X_{n k}\right) X_{n k}^{2} \mid \mathscr{F}_{n, k-1}\right)\right| \leqq \frac{1}{2} t^{2} V_{n n}^{2} \leqq \frac{1}{2} t^{2} C \text { a.s. }
$$


from (7). The convergence in (9) can thus be strengthened to the following:

(12) $\exp \left(-\sum_{k=1}^{n} E\left(g_{t}\left(X_{n k}\right) X_{n k}^{2} \mid \mathscr{F}_{n, k-1}\right)\right)$ converges to $\exp \left(-\int_{-\infty}^{\infty} g_{t}(x) d G(x)\right)$ in the mean of order 1 , as $n \rightarrow \infty$.

Now consider

$$
A_{n}=\exp \left[i t S_{n n}-\sum_{k=1}^{n} E\left(g_{t}\left(X_{n k}\right) X_{n k}^{2} \mid \mathscr{F}_{n, k-1}\right)\right]-1=\sum_{k=1}^{n} W_{n k},
$$

where

$$
\begin{aligned}
W_{n k}= & \left\{\exp \left[i t \sum_{j=1}^{k-1} X_{n j}-\sum_{j=1}^{k} E\left(g_{t}\left(X_{n j}\right) X_{n j}^{2} \mid \mathscr{F}_{n, j-1}\right)\right]\right\} \\
& \cdot\left\{\exp \left(i t X_{n k}\right)-\exp \left(E\left(g_{t}\left(X_{n k}\right) X_{n k}^{2} \mid \mathscr{F}_{n, k-1}\right)\right)\right\} .
\end{aligned}
$$

Therefore

$$
\begin{aligned}
E\left(W_{n k} \mid \mathscr{F}_{n, k-1}\right)= & \left\{Q\left(E\left(g_{t}\left(X_{n k}\right) X_{n k}^{2} \mid \mathscr{F}_{n, k-1}\right)\right)\right\} \\
& \cdot\left\{\exp \left[\left(i t \sum_{j=1}^{k-1} X_{n j}\right)-\sum_{j=1}^{k} E\left(g_{t}\left(X_{n j}\right) X_{n j}^{2} \mid \mathscr{F}_{n, j-1}\right)\right]\right\}
\end{aligned}
$$

where $Q(x)=e^{x}-1-x$, with

$$
|Q(x)| \leqq \frac{1}{2}|x|^{2} e^{|x|}
$$

Applying the inequalities (10), (7) and (14) to equation (13) gives

$$
\left|E\left(W_{n k} \mid \mathscr{F}_{n, k-1}\right)\right| \leqq \frac{1}{8} t^{4} \sigma_{n k}^{4} \cdot \exp \left(t^{2} C\right) .
$$

Therefore, since $\left|E A_{n}\right| \leqq E \sum_{k=1}^{n}\left|E\left(W_{n k} \mid \mathscr{F}_{n, k-1}\right)\right|$, we have

$$
\begin{aligned}
\left|E A_{n}\right| & \leqq \frac{1}{8} t^{4} \cdot \exp \left(t^{2} C\right) \cdot E \sum_{k=1}^{n} \sigma_{n k}^{4} \\
& \leqq \frac{1}{8} t^{4} \exp \left(t^{2} C\right) \cdot E\left(b_{n} V_{n n}^{2}\right) \\
& \rightarrow 0 \quad \text { as } n \rightarrow \infty
\end{aligned}
$$

because of (1) and the bound provided by (7). Because of (12) and (15), we can now complete the proof by applying the Lemma with $X_{n}=S_{n n}, f(t)=\phi(t)$, and

$$
W_{n}=Y_{n}(t)=\exp \left(\sum_{k=1}^{n} E\left(g_{t}\left(X_{n k}\right) X_{n k}^{2} \mid \mathscr{F}_{n, k-1}\right)\right),
$$

thus obtaining

$$
\lim _{n \rightarrow \infty} E \exp \left(i t S_{n n}\right)=\phi(t)
$$

for each fixed $t$.

CoRollaRY 1. If $V_{n n}^{2} \stackrel{p}{\rightarrow} \sigma^{2}$ (constant) as $n \rightarrow \infty$, and if

$$
\sum_{k=1}^{n} E\left(X_{n k}^{2} I\left(\left|X_{n k}\right| \geqq \varepsilon\right) \mid \mathscr{F}_{n, k-1}\right) \stackrel{p}{\rightarrow} 0 \text { as } n \rightarrow \infty,
$$

for all $\varepsilon>0$, then $S_{n n}$ converges in law as $n \rightarrow \infty$ to $N\left(0, \sigma^{2}\right)$. 
COROllaRY 2 (BROWN [3]). If $\left\{X_{n}, \mathscr{F}_{n}, n=1,2, \ldots\right\}$ is a martingale difference sequence with $S_{n}=X_{1}+\cdots+X_{n}, \sigma_{n}^{2}=E\left(X_{n}^{2} \mid \mathscr{F}_{n-1}\right), \quad V_{n}^{2}=\sum_{j=1}^{n} \sigma_{j}^{2}$, and $s_{n}^{2}=E S_{n}^{2}$ $=E V_{n}^{2}$ for $n=1,2, \ldots$; and if $s_{n}^{2} \uparrow \infty$ as $n \rightarrow \infty$,

$$
\begin{aligned}
& s_{n}^{-2} \max _{j \leqq n} \sigma_{j}^{2} \stackrel{p}{\longrightarrow} 0 \text { as } n \rightarrow \infty, \\
& V_{n}^{2} \stackrel{p}{\longrightarrow} \sigma^{2}(\text { constant }) \text { as } n \rightarrow \infty, \text { and } \\
& s_{n}^{-2} \sum_{j=1}^{n} E\left(X_{j}^{2} I\left(\left|X_{j}\right| \geqq \varepsilon s_{n}\right) \mid \mathscr{F}_{j-1}\right) \stackrel{p}{\rightarrow} 0 \text { as } n \rightarrow \infty,
\end{aligned}
$$

for all $\varepsilon>0$, then $S_{n} / s_{n}$ converges in law as $n \rightarrow \infty$ to $N\left(0, \sigma^{2}\right)$.

Although it simplifies matters somewhat, the assumption that the rows of the triangular array form a martingale difference sequence is not essential. As a trivial extension of Theorem 1, we can obtain

THEOREM 2. Let $U_{n 1}, U_{n 2}, \ldots, U_{n n}, n=1,2, \ldots$, be a triangular array of r.vs. with $X_{n j}=U_{n j}-E\left(U_{n j} \mid \mathscr{F}_{n, j-1}\right), j=1,2, \ldots, n ; n=1,2, \ldots$, such that $X_{n 1}, \ldots, X_{n n}$, $n=1,2, \ldots$, form a martingale elementary system (i.e. that (1) and (2) hold). If equation (3) holds and if $\sum_{k=1}^{n} E\left(U_{n k} \mid \mathscr{F}_{n, k-1}\right) \stackrel{p}{\rightarrow} \gamma$ (constant) as $n \rightarrow \infty$, then $T_{n n}=U_{n 1}+\cdots+U_{n n}$ converges in law as $n \rightarrow \infty$ to an infinitely divisible r.v. with ch.f. $\phi(t)$ given by

$$
\log \phi(t)=i t \gamma+\int_{-\infty}^{\infty}\left(e^{i t x}-1-i t x\right) x^{-2} d G(x)
$$

\section{REFERENCES}

1. G. M. Bavli, Über einige Verallgemeinerungen der Grenzwertsätze derWahrscheinlichkeitsrechnung, Mat. Sb. 1 (43) (1936), 917-930.

2. Patrick Billingsley, The Lindeberg-Lévy theorem for martingales, Proc. Amer. Math. Soc. 12 (1961), 788-792. MR 23 \#A4165.

3. B. M. Brown, Martingale central limit theorems, Ann. Math. Statist. 42 (1971), 59-66.

4. B. V. Gnedenko, Course in the theory of probability, 2nd ed., GITTL, Moscow, 1950; English transl., Chelsea, New York, 1962. MR 13, 565.

La Trobe University, Bundoora, Victoria, Australia

University of Sydney, Sydney, N.S.W., Australia 